\title{
N $86-19929$
}

Application of Photosynthetic $\mathrm{N}_{2}$-fixing Cyanobacteria to the CELSS Program

by Lester Packer, Ian Fry, and Shimshon Belkin, Membrane Bioenergetics Group, Applied Science Division, Lawrence Berkeley Laboratory, University of California, Berkeley, CA 94720

\section{Abstract}

Certain cyanobacterial species have the unique ability to reduce atmospheric $\mathrm{N}_{2}$ to organic nitrogen. These organisms combine the ease of cultivation characteristic of prokaryotes with the fully developed photosynthetic apparatus of higher plants. This, along with their ability to adapt to changes in their environment by modulation of certain biochemical pathways, make them attractive candidates for incorporation into the CELSS program.

A high percentage of the cyanobacterial biomass is in the form of protein, much of which exists in discrete high molecular weight granules (phycobilisomes and carboxysomes). We have shown that with proper manipulation of the osmotic environment, high amounts of granular glycogen can also be produced. These particular fractions, along with the ability of certain species to aggregate, may negate future need for conventional harvesting and fractionation techniques, such as high speed centrifugation.

We are undertaking a study, using commercially 


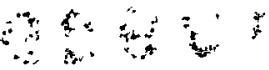

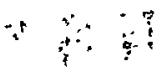

available air lift fermentors, to simultaneously monitor biomass production, $\mathrm{N}_{2}$-fixation, photosynthesis, respiration, and sensitivity to oxidative damage during growth under various nutritional and light regimes, to establish a data base for the integration of these organisms into a CELSS program.

\section{Introduction}

The use of biological components in the CELSS program as subsystems for the revitalizaton of air, waste processing, and for the production of food has been proposed for long-term space flight ${ }^{(1)}$. To this end, many systems of biomass (protein, carbohydrate, lipid) production are under consideration by NASA. Employment of photosynthetic systems (higher plants, green algae and cyanobacteria) allow biomass production from relatively simple components which are readily recycled in a CELSS system, namely $\mathrm{CO}_{2}$ ' minerals $\left(\mathrm{NO}_{3}^{-}, \mathrm{PO}_{4}^{-}, \mathrm{K}^{+}, \mathrm{Na}^{+}\right.$, etc.) and micronutrients.

The production of plant material in a closed system presents several problems, two of which we shall address here. A primary consideration is that of the energy requirement for continuous operation, including illumination, temperature control and various maintenance mechanisms. One way in which the energy demands can be lessened is by modifying the quality of the light used. Illumination of photosynthetic systems with white light 
(300-700nm range) is wasteful, since photosynthetic organisms utilize only selected regions of the visible spectrum. Tailoring the emission profile of the light source to match that of the action spectrum of photosynthesis, or to select an "emission band" in a region of maximal absorption by some of the photosynthetic pigments would present an energy saving, especially if the selected "emission band" was in the blue region, when using fluorescent light sources.

A second concern is the probability of nitrogen loss from the closed system by the action of contaminating denitrifying bacteria, which degrade $\mathrm{NO}_{3}^{-}$to $\mathrm{N}_{2}$ gas, which is then lost from the biological system ${ }^{(2)}$. Denitrification is most likely to occur irrespective of the method of waste processing due to the storage of human waste and non-food biomass (cellulose), which would result in an irreversible loss of biologically essential nitrogen.

To address the above problems, we have proposed the use of cyanobacteria (blue-green algae) as the photosynthetic organism of choice.

Cyanobacteria occupy a unique position in the hierarchy of plant form and structure. Like higher plants, they carry out the $\mathrm{O}_{2}$ evolving photolytic cleavage : of water and the subsequent fixation of $\mathrm{CO}_{2}$ into carbohydrate. Unlike higher plants, however, during periods of 
nitrogen starvation (when $\mathrm{NO}_{3}^{-}$levels in the medium are depleted) certain species differentiate specialized cell types (heterocysts) which maintain low oxygen tension, a prerequisite for their major function: the reduction of atmospheric $\mathrm{N}_{2}$ to $\mathrm{NH}_{3}$, prior to its incorporation into amino acids and proteins. In addition, cyanobacteria possess prokaryotic genetics, which allow the future possibility of genetic manipulation. Also, environmental factors have been shown in certain strains to modify the partitioning of photosynthetic reductant to certain macromolecules $(3,4)$ and storage carbohydrate ${ }^{(5)}$. These factors make the cyanobacteria prime candidates for consideration in the CELSS program, as either a primary source of biomass or as a supplement to other algal/higher plant subsystems, to reverse the effect of denitrifying bacteria, and recycle atmospheric $\mathrm{N}_{2}$ back into the biological systems.

\section{Research Objectives}

Due to the suitability of cyanobacteria for integration as a CELSS subsystem, we propose to investigate biomass production in the form of dietary compatible macromolecules (protein, lipid, carbohydrate) of nitrogen fixing heterocystous cyanobacteria in (commercially available) air-lift type fermentors. The research plan is to investigate environmental effects of nutrient supply, particularly $\mathrm{CO}_{2}$ (the major limiting factor for plant 
growth) and the effects of various light regimes on productivity, in particular where it affects $\mathrm{N}_{2}$ fixation. Due to the more efficient conversion of UV photons to photons in the blue region rather than to the full visible range by fluorescent discharge tubes, we shall investigate growth characteristics, biomass production, and certain relevant biochemical functions (photosynthetic $\mathrm{O}_{2}$ evolution, respiration, and nitrogen fixation) on cells grown under blue light, compared to an equal concentration of white light photons. The blue "emission band" overlaps absorption bands of the light harvesting antennae pigments chlorophyll and phycobiliproteins.

One of the major factors affecting nitrogenase activity is the ambiant oxygen level. Although the heterocysts possess a thick cell wall which slows $\mathrm{O}_{2}$ diffusion to rates that are compatible with the rates of $\mathrm{O}_{2}$ removal by the respiratory system, it is quite likely that intracellular levels of $\mathrm{O}_{2}$ (particularly in the cells directly adjoining the heterocysts) may exceed usual environmental levels. To determine the role of $\mathrm{O}_{2}$ in such a system, we have been developing an electron spin resonance (ESR) method which monitors the broadening of a paramagnetic spin probe molecule by interaction with $\mathrm{O}_{2}$, which is itself a paramagnet in the ground state. Interaction of the spin probe with other paramagnetic species causes a measurable broadening of the probe's ESR signal line width. Since the probe can be completely broadened 
by adding a cell membrane impermeable paramagntic reagent, the broadening due to $\mathrm{O}_{2}$ inside of the cell can be monitored. Using this technique, we plan to investigate the effect of cellular $\mathrm{O}_{2}$ levels, where this may influence the rates of nitrogen fixation.

\section{Materials and Methods}

Nostoc muscorum (Anabaena \#7119) was grown in BGll medium minus nitrate in a 2 litre Bethesda Research Laboratories Airlift Fermentor at $28^{\circ} \mathrm{C}$, $150 \mathrm{u} \mathrm{Es}^{-1} \mathrm{~m}^{-2}$ light (using Bethesda Research Laboratories 2201 LB day light white $300-700 \mathrm{~nm}$, or $2201 \mathrm{LF}$ blue light $400-500 \mathrm{~nm}$ ) with an air flow rate of 2 litres/min, supplemented with $0.5 \% \mathrm{CO}_{2} \cdot 200 \mathrm{ml}$ aliquots were withdrawn daily, the fermentor volume made up by addition of $200 \mathrm{ml}$ of sterile medium. Cells were centrifuged at $10,000 \mathrm{xg} / 10 \mathrm{~min}$ and resuspended to $10 \mathrm{ml}$ in $\mathrm{BG} 1 \mathrm{l}$ medium suplemented with $10 \mathrm{mM}$ Tes buffer $\mathrm{pH} 7.0$.

$\mathrm{O}_{2}$ evolution was monitored polarographically in BGIl plus lomM Tes pH7.0 with a cell density equivalent to $1-2$ ug chlorophyll/ml. Nitrogenase activity was determined in whole filaments by monitoring acetylene reduction using a Varian Model 3700 gas chromatograph fitted with a Poropak T column. Cells ( 3 ug chlorophyll in $3 \mathrm{ml}$ ) were assayed in a $5 \mathrm{ml}$ vial under air plus $10 \%(\mathrm{v} / \mathrm{v}) \mathrm{C}_{2} \mathrm{H}_{2}$ in a shaking 
Table I

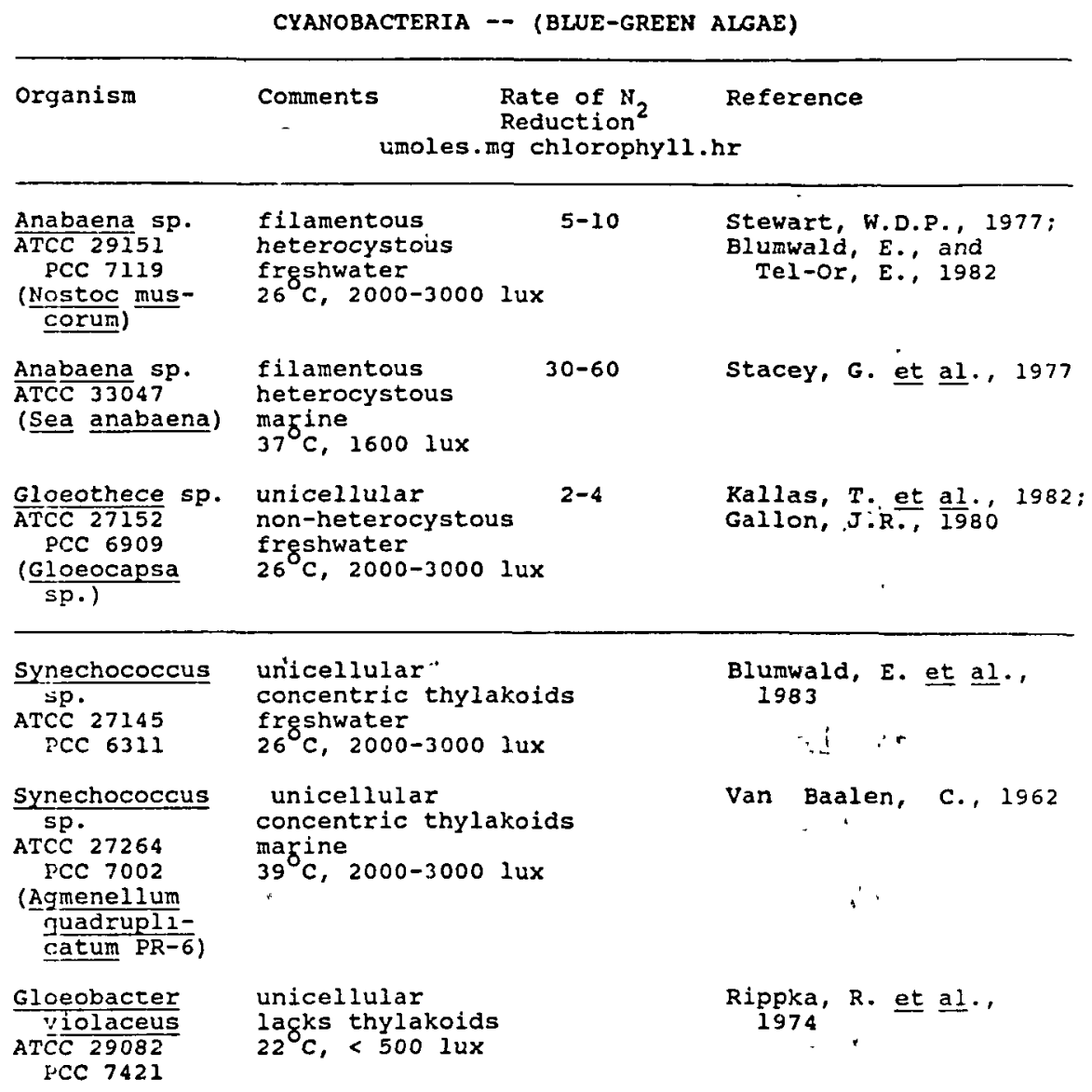

\section{Table II}

CELL COMPOSITION OF NOSTOC MUSCORUM GROWN UNDER WHITE (300-700nm) AND BLUE (400-500 nm) IIGHT

percent Dry Weight

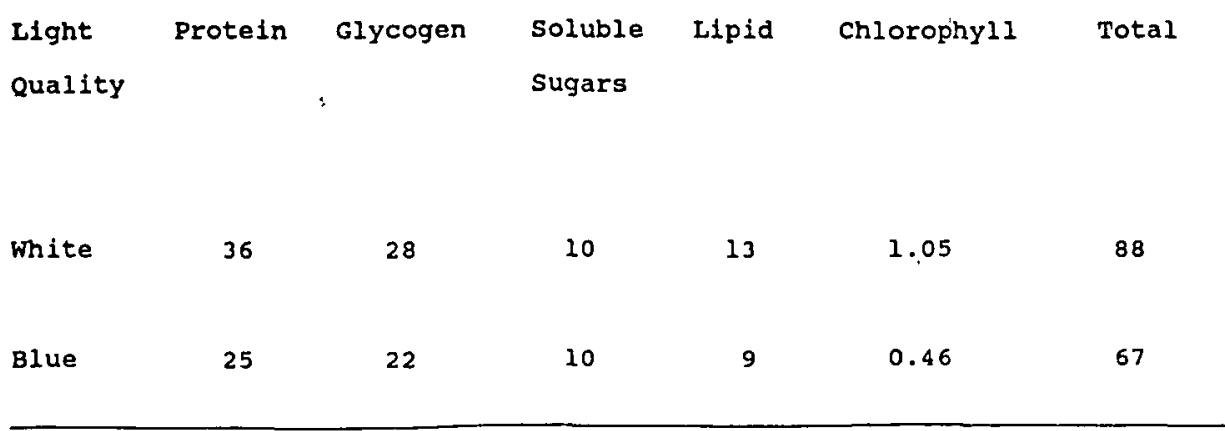


waterbath at $28^{\circ} \mathrm{C}$ under $50 \mathrm{uEs}^{-1} \mathrm{~m}^{-2}$ blue or white light.

Light intensities were measured using a Li-Cor inc. Integrating quantum/radiometer/photometer Li-188B, with a Li-190SB quantum sensor.

\section{oxygen-broadening experiments:}

Dense suspensions (1-2 $\mathrm{mg} \mathrm{chl} / \mathrm{ml})$ of the cyanobacterium Agmenellum quadruplicatum, in ASP-II medium, were introduced into 75 ul capillary tubes, together with 0.5 $\mathrm{mM}$ of the nitroxide spin-probe 3 -hydroxy-2,2,5,5-tetramethyl 3-pyrrolin-l-oxyl (PCA-ol). The ESR signal was monitored by using a varian El09 EPR spectrometer. The sample was illuminated inside the cavity with an oriel Universal Xenon Air Lamp.

\section{Experimental Results}

Representative strains of cyanobacteria are shown in Table I, along with their nitrogen fixing capabilities and growth conditions. The heterocystous filamentous strain Nostoc muscorum (Anabaena 7119) was chosen for the present study.

Growth of Nostoc muscorum under blue (400-500nm) and white (300-700nm) light at $150 \mathrm{uE} / \mathrm{s} / \mathrm{m}^{2}$, is shown in Figure 1. Clearly the cells grow readily under the blue light, 
Fig. 1) Growth of Nostoc muscorum under white $(300-500 \mathrm{~nm})$ and blue $(400-500 \mathrm{~nm})$ light, measured by absorbance changes at $550 \mathrm{~nm}$. $G=c e l l$ doubling time.

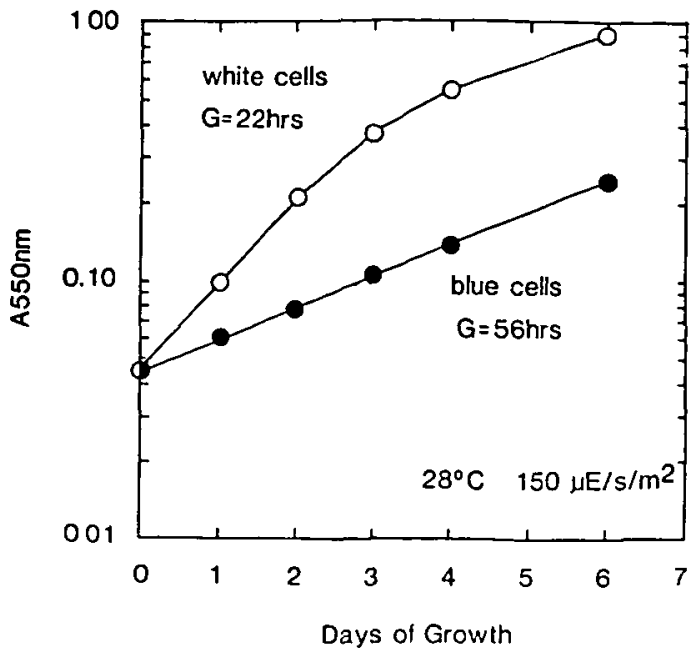

even though the cell doubling time (generation time, G) is increased by a factor of 2.5. Analysis of photosynthetic $\mathrm{O}_{2}$ evolution and nitrogenase activity during growth under blue light reveal very little difference from those grown under white light when rates were calculated on a total protein basis (Figure 2 and 3, upper sections). Calculation of rates (particularly photosynthesis) on a total chlorophyll basis reveal an apparent increase in photosynthetic rate (Figure 2 , lower section). However, this reflects a decrease in total cell chlorophyll, a chromatic adaption to blue light (see Table II). Analysis of cellular products after a seven day growth period under blue and white light reveals that little change occurs in soluble sugars, glycogen, or lipids, while total protein decreases only by $10 \%$ (Table II). An "unknown" component which does increase (see "Total" column in Table II), is probably cell wall material, and is under further characterization. 


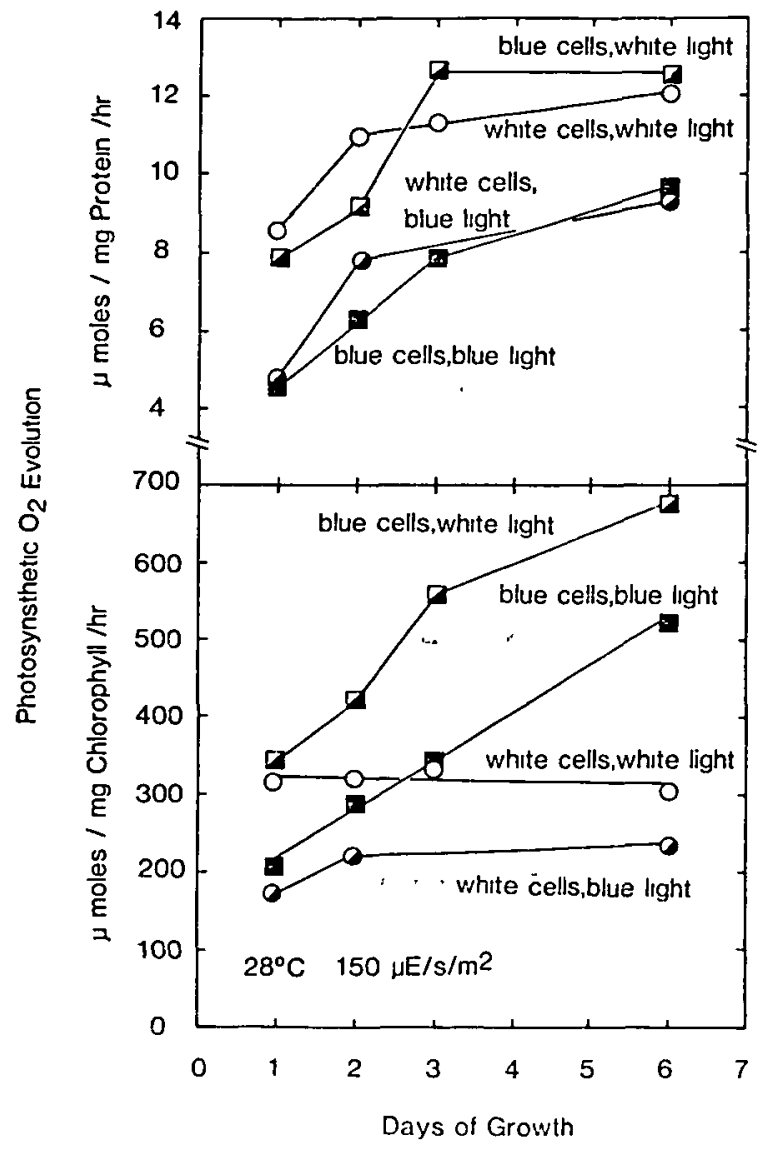

Fig. 2) Photosynthetic $\mathrm{O}_{2}$ evolution of cells grown ${ }^{2}$ under white or blue light, based on total protein (upper secion) and chlorophyll (lower section) conditions as in Materials and Methods.

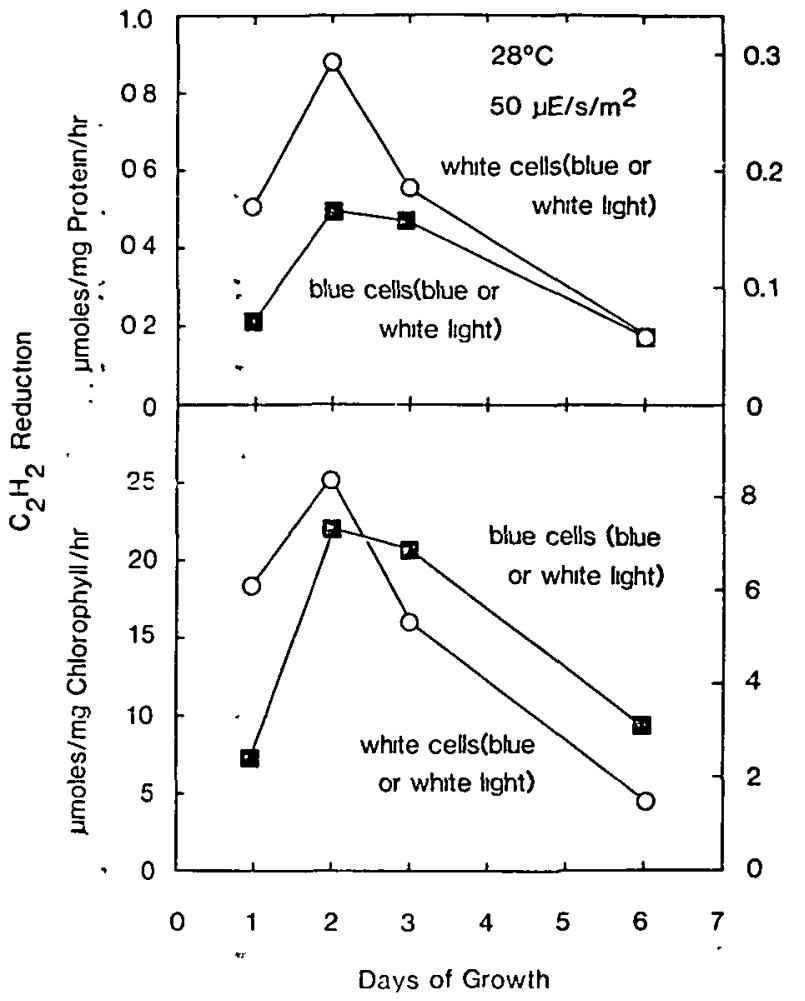

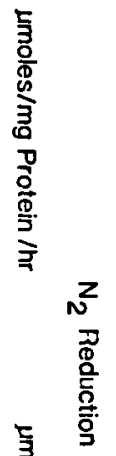

Fig. 3) -Nitrogenase activity, as measured by acetylene reduction based on total protein (upper section) and chlorophyll (lower section) conditions as in Materials and Methods.

In conclusion, the nitrogen fixing strain Nostoc muscorum is capable of growth under light at the blue end of the visible spectrum. One must evaluate whether the energy saving gained by employing blue photons.outweigh the slower growth rate (and hence biomas's production). However, if a system employing a continuous culture (kept at a fixed point in the exponential growth phase, linear section of Figure 1) for air revitalization, etc., then a slower growth rate may be advantageous, requiring less addition of growth medium to maintain the constant cell 
density.

Nitrogenase, the enzyme responsible for the biological fixation of atmospheric $\mathrm{N}_{2}$, is extremely oxygensensitive(2). One of the modes in which cyanobacteria "solved" the problem of $\mathrm{N}_{2}$-fixation under aerobic conditions was the development of the heterocysts mentioned above. However, as with other nitrogenase containing microorganisms, the mechanisms by which these cells retain an oxygen-free internal environment is not completely understood.

We have recently developed an ESR technique which enables us to measure introcellular $\mathrm{O}_{2}$ concentrations. We hope to utilize this method to gain a better understanding of the protection mechanisms against the toxic effects of $\mathrm{O}_{2}$ to nitrogenase, and thereby of the ways the activity of this enzyme may be manipulated to the project's advantage.
A. Origınal Signal
B. 2nd Harmonic
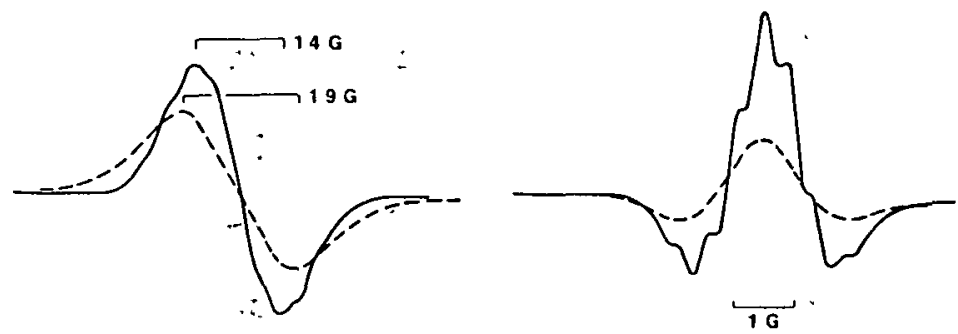

Fig. 4) The center ESR signal of PCA-Ol (A) and the 2nd derivative (B), before (solid line) and after (dashed line) 1 min illumination of A. quadruplicatum suspension (lmg chl/ml). PCA-ol concentration was $0.5 \mathrm{mM}$. 
The above technique is based upon the broadening effect dissolved $\mathrm{O}_{2}$ has on the nitroxide spin probes ESR signal. An example of such an effect is presented in Figure 4. Photosynthetically-generated oxygen, in a suspension of the cyanobacterim Agmenellum quadruplicatum, causes a broadening of the signal by $0.5 \mathrm{G}$ (Figure $4 \mathrm{a}$ ). The effect is even more pronounced when a higher derivative of the spectrum is examined (Figure $4 \mathrm{~b}$ ). According to our calculations, this broadening is equivalent to $\mathrm{O}_{2}$ concentration in the suspension of $2.5 \mathrm{mM}$. Furthermore, by quenching the extracellular signal, it is possible to examine only the probe inside the cells (Figure 5), and using the measured degree of broadening calculate intra-

Fig. 5) A schematic presentation of the mode by which an internal spinprobe signal is differentiated from the external one.

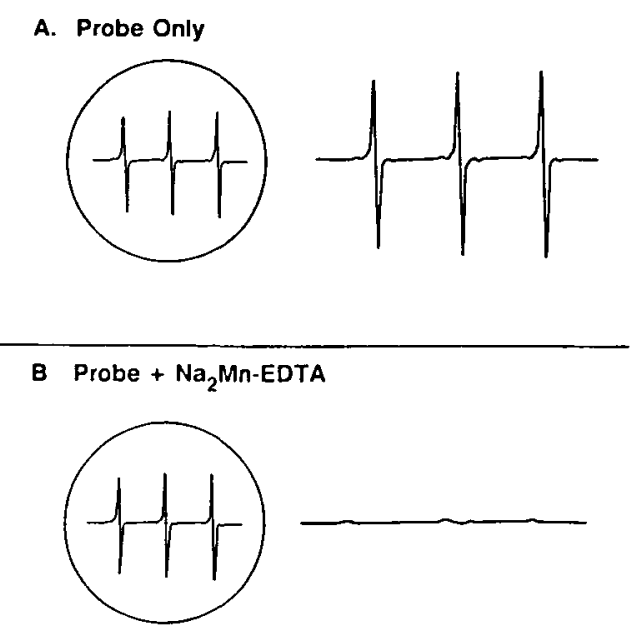

cellular $\mathrm{O}_{2}$ concentrations. Studies are presently underway to compare $\mathrm{O}_{2}$ levels in non- $\mathrm{N}_{2}$-fixing and some $\mathrm{N}_{2}-$ fixing cyanobacterial cells, as well as to establish the $\mathrm{O}_{2}$-sensitivity of various photosynthetic activities. 


\section{Future Research}

We propose to continue the investigation of the effects of selected wavelengths (and combinations of selected wavelengths) on biomass production and the biochemical functions of $\mathrm{O}_{2}$ evolution and $\mathrm{N}_{2}$ fixation.

In non-nitrogen fixing strains of cyanobacteria we have shown that changes in the environmental conditions, particularly increases in salinity, result in a marked increase in soluble sugars $(6,7)$ and carbohydrate storage products $^{(5)}$. We plan to investigate the partitioning of photosynthetically derived reductant between the various macromolecules (protein, lipid, carbohydrate) under such conditions to determine the ability to manipulate the relative levels. of the various macromolucules by environmental changes. 


\section{Referènces}

1. Macelroy, R.D., and Bredt, J. (1984) NASA CP-2378, XXV COSPAR Conference, Graz, Austria (MacElroy, Smernoff and Klein, ed.s) pp 1-9.

2. Payne, W.J., Rowe, J.E. and Sherr, B.F. (1980) Ih; Nitrogen Fixation vol 1 (W.E. Newton and W.H. OrmeJohnson, ed.s) pp 29-42 Vain Park Press, Baltimore

3. Reed, R.H., Richardson, D.L., Warr, S.R.C. and stewart (1984) Journal of Genetic Microbiology $130,1-4$

4. Mackay, M.A., Norton, R.S. and Borowitzka, L.J. (1984) Journal of Genetic Microbiology 130, 2177-2191

5. Fry, I.V., Huflejt, M., Erber, W.W.A., Peschek, G.A. and Packer, L. (1985) Submitted for publication

6. Blumwald, E., Mehlhorn, R.J. and Packer, L. (1983) Plant Physiology 73, 377-380

7. Blumwald, E., Mehlhorn, R.J., and Packer, L. (1983) Proc. National Academy of Science 80, 2599-26̣02 